\title{
Religiosidade/Espiritualidade na Prática Clínica: Círculo Vicioso entre Demanda e Ausência de Treinamento
}

\author{
Juliana Schmidt Raddatz, ${ }^{1,2}$ \\ Roberta Fin Motta ${ }^{1}$ \\ Letícia Oliveira Alminhana ${ }^{1,2}$ \\ ${ }^{1}$ Pontifícia Universidade Católica do Rio Grande do Sul, Porto Alegre, RS \\ ${ }^{2}$ Instituto Unusual in Psychology RS
}

\begin{abstract}
Resumo
Diante do crescente número de pesquisas sobre Religiosidade e Espiritualidade (R/E), este artigo objetiva analisar a percepção de profissionais da saúde em relação a esses temas. Foram realizadas entrevistas semiestruturadas, coletadas nos locais de trabalho de seis profissionais liberais, utilizando a Análise de Conteúdo de Bardin, na abordagem qualitativa. Seis categorias emergiram das análises qualitativas: 1. R/E: fusão de conceitos e conexão com o transcendente; 2 . Espiritualidade como facilitadora; 3. Demanda aparece informalmente; 4. Falta de treinamento; 5. Na prática é diferente; 6. A temática nos currículos acadêmicos. Os resultados indicaram que os profissionais observam a relevância da temática, embora ela apareça apenas de forma implícita. Como um círculo vicioso, a ausência de treinamento adequado pode levar à falta de inserção do tema na formação acadêmica. Futuros profissionais precisam receber treinamento para saber como acolher a religiosidade e a espiritualidade de pacientes/clientes de forma ética, visando a promoção de saúde.

Palavras-chave: religiosidade/espiritualidade, prática clínica, profissionais de saúde
\end{abstract}

\section{Religiosity/Spirituality in the Clinical Practice: Vicious Cicle between Demand and Lack of Training}

\begin{abstract}
With the growing number of researches on religiousness and spirituality, this article aimed to analyze the perception of health professionals in relation to these themes. Semi-structured interviews were conducted at the workplace of six professionals using Bardin's Content Analysis, in the qualitative approach. Six categories emerged from the qualitative analyses: 1. Religiousness and Spirituality: fusion of concepts and connection with the transcendent; 2. Spirituality as a facilitator; 3. Demand appears informally; 4. Lack of training; 5. In practice it is different; 6 . The theme in academic curricula. The results indicated that professionals observe the relevance of the theme, although it appears only implicitly. As a vicious circle, the absence of adequate training can lead to the lack of insertion of these subjects into academic training courses. Future professionals need to receive training to know how to welcome patients/clients religiousness and spirituality with ethics, seeking health promotion. Keywords: religiosity/spirituality; clinical practice; health professional
\end{abstract}

\section{Religiosidad/Espiritualidad en la Practica Clínica: Circulo Vicioso entre Demanda y Ausencia de Entrenamiento}

\section{Resumen}

Ante el creciente número de investigaciones sobre religiosidad y espiritualidad R/E, este artículo se propone analizar la percepción de los profesionales de salud con relación a estos temas. Se realizaron entrevistas semiestructuradas, recogidas en los lugares de trabajo de seis profesionales liberales utilizando el Análisis de Contenido de Bardin, con un enfoque cualitativo. De estos análisis surgieron seis categorías: 1. Religiosidad y Espiritualidad: fusión de conceptos y conexión con lo trascendente; 2. Espiritualidad como facilitadora; 3. Demanda aparece informalmente; 4. Falta de entrenamiento; 5. En la práctica es diferente; 6. Temática en currículos académicos. Los resultados indicaron que los profesionales observaron la importancia del tema, aunque sólo se señale de forma implícita. Como un círculo vicioso, la falta de entrenamiento adecuado puede llevar a la falta de inserción del tema en la formación académica. Los futuros profesionales necesitan ser entrenados para saber como acoger la religiosidad y espiritualidad de pacientes / clientes de forma ética, con el objetivo de promover la salud.

Palabras clave: religiosidad/espiritualidad; práctica clínica; profesionales de la salud

\section{Introdução}

As pesquisas sobre espiritualidade e saúde vêm crescendo ao longo do tempo (Farias, Underwood, \& Claridge, 2013; Koenig, King, \& Carson, 2012; Lucchetti, Peres, Vallada, \& Lucchetti, 2015; Martins \& Zangari, 2012; Parra \& Espinosa Paul, 2010). No Brasil, existem muitos estudos publicados e núcleos de pesquisa formados para estudar a temática (Dalgalarrondo, 2007; Menezes Jr., Alminhana, \& Moreira-Almeida, 2012; Moreira-Almeida et al., 2006; Moreira-Almeida, Neto, \& Greyson, 2007), não só na Psicologia (Machado, Zangari, Maraldi, Martins, \& Shimabucuro, 2017; Campos \& Ribeiro, 2017; Oman \& Lukoff, 2018), como na 
Enfermagem, tanto na clínica quanto na graduação, (Siqueira, Cecagno, Medeiros, Sampaio, \& Rangel, 2017; Simões, Martins, dos Santos, Santana, \& Pilger, 2018 ), na Fisioterapia (Nepomuceno, Almeida, Fonseca, \& Vieira, 2018; Neto \& Paiva, 2016) e em outras áreas como a Psiquiatria, por exemplo (Alminhana, Ramos, Nardini, \& Marques, 2017; Arruda, Marafanti, da Silva, Pinheiro, Shiozawa, \& Cordeiro, 2018; VanderWeele, Balboni, \& Balboni, 2018; Lavorato-Neto, Rodrigues, Turato, \& Campos, 2018). Em uma publicação recente sobre o assunto, a Associação Mundial de Psiquiatria (WPA) divulgou um Position Statement, declaração sobre a importância da Religiosidade/Espiritualidade (R/E) para o ensino, prática, clínica e pesquisa em psiquiatria (Moreira-Almeida et al., 2016).

Há algumas décadas, o $G W$ Institute for Spirituality and Health Care da George Washington University/ EUA tem oferecido programas de treinamento para médicos e profissionais da saúde em geral no intuito de incluir questões relacionadas à espiritualidade, crenças ou fé ao atendimento dos pacientes. De acordo com sua fundadora, Puchalski (2001), os pacientes costumam fazer perguntas como: "Por que isto está acontecendo comigo agora? Existe um Deus? Caso exista, ele estará lá quando eu morrer?" entre outras. Para a autora, as pessoas esperam que seus médicos e cuidadores sejam capazes de ouvi-las e de oferecer suporte a elas. Pargament, Koenig, \& Perez, (2000; 1998), também chamam a atenção para o fato de que as pessoas costumam fazer uso de sua R/E como estratégia para enfrentar o estresse. Tal estratégia, denominada de Coping Religioso/Espiritual, pode apresentar benefícios ou malefícios para o decorrer do tratamento dos pacientes e cabe aos profissionais estarem abertos e atentos a todas essas questões.

\section{Religiosidade e Espiritualidade: Definições Principais}

Segundo Lucchetti, Granero, Bassi, Latorraca e Nacif (2010), a religiosidade pode ser definida como a maneira com que o indivíduo acredita e segue a sua religião podendo ser com participação organizacional em templo religioso ou de forma mais pessoal, como orações, leituras e programas de televisão religiosos. A religiosidade pode ser vista também como o enaltecimento de alguma prática religiosa, no sentido de valorização da experiência, não estando ligada necessariamente a nenhuma religião específica.

Por outro lado, a espiritualidade abrange a instância pessoal e existencial, sendo assim, menos relacionada diretamente a instituições, rituais e dogmas
(Koenig et al., 2012). Koenig (2005), entretanto, sugere que a espiritualidade é entendida como uma relação com o sagrado e o transcendente, um poder supremo, e pode ou não estar relacionada a uma religião. Outros autores definem a espiritualidade como a busca inerente da espécie humana em conexão com algo maior que si próprio, algo que agregue sentido em sua existência (Saad, Masiero, \& Battistella, 2016; Moreira-Almeida, Lucchetti, 2016, Kørup et al., 2018).

Ainda que não haja um consenso científico para elucidar especificamente cada categoria, por se tratar de um campo intensamente subjetivo, alguns autores fazem essa diferenciação para fins didáticos e de pesquisa. Importante salientar que, na abordagem clínica, $\mathrm{R} / \mathrm{E}$ não podem ser pré-definidas pelo profissional. Cabe a este unicamente acolher o sentido que esta questão possui do ponto de vista do paciente/cliente (Lucchetti et al., 2010; Puchalski, 2001).

Apesar das evidências sobre o impacto das crenças religiosas/espirituais na saúde, ainda existe uma lacuna entre os profissionais e seus pacientes no que tange a abordagem do tema (Dein, Cook, Powell, \& Eagger, 2010; Harold Koenig et al., 2012). Ainda que alguns considerem tal abordagem inapropriada, é preciso observar o quanto deixar de ouvir os pacientes em relação às suas crenças pode acarretar em uma falha no objetivo central de compreender as pessoas de forma abrangente e contextualizada (Dein et al., 2010). Um estudo conduzido em um hospital oncológico de São Paulo entrevistou 11 profissionais da saúde perguntando sobre a sua espiritualidade e a de seus pacientes (Espíndula, Valle, \& Bello, 2010). De acordo com as análises qualitativas, apesar da maneira pragmática e naturalista de ver o mundo, consequente da formação científica recebida, os profissionais parecem ressignificar suas próprias crenças com o contato da religiosidade de seus pacientes. Além disso, sua principal preocupação é que seus pacientes acessem sua R/E sem, contudo, perder o senso de realidade.

Outro estudo realizado no Brasil, envolvendo 12 faculdades de Medicina e 5950 estudantes mostrou que, em sua maioria, os alunos acreditavam que a religiosidade/espiritualidade impacta a saúde das pessoas (Lucchetti, de Oliveira, Koenig, Leite, \& Lucchetti, 2013). A maioria gostaria de poder abordar a temática em sua prática clínica, embora a metade ainda se sinta despreparada para fazê-lo.

Ainda sobre a lacuna no aprendizado, outro estudo feito com 120 estudantes de enfermagem de uma universidade de São Paulo, buscou identificar a 
compreensão desses estudantes em relação a saúde e espiritualidade. Os autores investigavam se o entendimento sobre espiritualidade e religiosidade teria sido abordado durante a graduação. Como resultado, foi observado que, 76\% dos alunos entrevistados acreditam que a espiritualidade influencia na saúde, e 54\% apontaram que a formação universitária não oferece informações suficientes para que se sintam preparados em abordar o tema com os pacientes quando necessário (Espinha, Camargo, Silva, Pakvequeires, \& Luchetti, 2013). Assim sendo, observa-se a carência de treinamento, bem como o gap existente entre as expectativas dos estudantes de Medicina e a realidade do currículo das universidades brasileiras.

Percebendo a importância do tema, a lacuna no aprendizado e em pesquisas sobre o assunto, e o quanto esse tópico ainda está distante da prática do profissional da saúde, o presente estudo buscou observar fenomenologicamente como os profissionais de saúde percebem e tratam a $\mathrm{R} / \mathrm{E}$ em seu dia a dia com seus pacientes. Profissionais de seis áreas da saúde foram questionados em relação aos conceitos de $\mathrm{R} / \mathrm{E}$; à percepção da demanda por parte dos pacientes e à formação e treinamento na área. Em última instância, o estudo pretendeu ir além da descrição das associações entre R/E e saúde, discutindo o passo seguinte, a saber, como tais questões impactam a prática diária dos atendimentos em saúde.

\section{Método}

Esta foi uma pesquisa qualitativa, de caráter exploratório-descritivo desenvolvida, em uma cidade brasileira do interior do estado do Rio Grande do Sul. Foram entrevistados seis profissionais de saúde: um odontólogo, um médico, um psicólogo, um técnico de enfermagem, um fisioterapeuta e um enfermeiro, onde três deles eram profissionais liberais e o restante da amostra trabalhava em regime CLT. Os profissionais não eram membros de uma mesma equipe e atendiam em sistema majoritariamente privado. Os participantes foram quatro mulheres e dois homens, com média de idade de 40,8 anos e desvio padrão de 7,57. Em relação ao estado civil, a amostra se distribuiu em cinco casados e um solteiro. Todos se autodefiniram como profissionais liberais, sendo que quatro eram pós-graduados. Em relação à religiosidade, dois se declararam católicos, três espíritas e um sem religião. Os critérios de inclusão: ser maior de 18 anos, profissional de alguma área da saúde com exercício da sua profissão por dois anos ou mais.
O local primário de recrutamento dos profissionais foi uma sala previamente selecionada na instituição de ensino da pesquisadora. Em cada entrevista, foi pedido para que o profissional indicasse outro colega da área da saúde que se propusesse a fazer parte da pesquisa. Foi adotado o critério de saturação para composição da amostra. Os entrevistados foram selecionados por conveniência e "bola de neve" por meio de indicações de outros profissionais e incluídos no estudo por apresentarem disponibilidade para responder à entrevista. Os participantes receberam a sigla "P" acrescida de um numeral para não serem identificados.

\section{Instrumentos}

- Entrevista sociodemográfica: a) sexo; b) nível educacional; c) situação conjugal atual; d) ocupação; e) perfil religioso, e f) formação prévia sobre $\mathrm{R} / \mathrm{E}$ e Saúde.

- Entrevista semiestruturada: $\mathrm{Na}$ entrevista foram propostas oito questões semiabertas, em que os profissionais poderiam falar um pouco sobre suas experiências. Os critérios utilizados para maior investigação de uma pergunta disparadora foram os estudos sobre espiritualidade e religiosidade na prática clínica, como segue: a) O que é espiritualidade? b) O que é religiosidade? c) Há demanda em relação à espiritualidade no contexto terapêutico? E como essa demanda aparece? d) Como é feito o manejo do tema com o paciente? e) Você percebe a espiritualidade como facilitadora ou não no processo saúde/doença? f) Você se sente preparada (o) ou capacitada(o) para manejar o tema quando ele aparece? g) Na sua formação, você teve acesso ao tema espiritualidade de alguma forma? h) Caso você ache importante, quais as possibilidades para se ter mais acesso ao tema na formação?

\section{Procedimentos para Coleta de Informações}

As entrevistas foram realizadas individualmente no local de trabalho de todos os sujeitos e duraram aproximadamente 40 minutos. Em todas as abordagens, as entrevistas foram precedidas de explicação e esclarecimentos sobre o objetivo da pesquisa e forma de coleta dos dados, bem como sobre os procedimentos éticos. Após a leitura e a assinatura do Termo de Consentimento Livre e Esclarecido (TCLE), as entrevistas foram gravadas em áudio, depois de transcritas, desgravadas. Para preservar o anonimato e o sigilo, foram adotados números como modo de identificação dos participantes. O estudo foi aprovado por Comitê de Ética em 
Pesquisa e a Resolução 466/12 do CNS foi respeitada com o número CAEE 46585415.0.0000.5306.

\section{Análises}

As informações coletadas foram submetidas à análise de conteúdo proposta por Bardin (2010). Segundo a autora, essa forma de investigação é definida por um conjunto de técnicas de análise que visa obter categorias de discurso. Por meio de procedimentos sistemáticos e de descrição do conteúdo das mensagens, é possível observar indicadores nas respostas que permitem inferir conhecimentos relativos às condições de produção e de recepção dessas mensagens. Foram seguidos os seguintes passos da análise de Bardin: a pré-análise, onde foi feita a coleta dos depoimentos dos entrevistados através da gravação e transcrição das respostas; a análise exploratória dos dados transcritos; a interpretação dos dados realizada por dois juízes (JR e LOA); bem como a categorização dos temas elencados juntamente com a fundamentação teórica. A análise feita pelos dois juízes obteve um nível adequado e 95\% de concordância (utilizando o índice de concordância de Kappa).

\section{Resultados e Discussão}

Este estudo buscou explorar as percepções dos profissionais da saúde acerca do tema $\mathrm{R} / \mathrm{E}$ em suas práticas de trabalho. Assim, foi possível observar que os participantes começam apontando uma demanda que aparece em conversas informais e que é vista como facilitadora do processo de saúde. No entanto, os profissionais não se sentem aptos para abordar a questão em sua prática e não veem possibilidade de inserir o tema Religiosidade/Espiritualidade na academia. Em consequência disso, todos os eixos observados, neste estudo, tem como pano de fundo a falta de uma discussão mais crítica acerca do tema, no meio científico e na formação acadêmica. Em uma perspectiva ampla, os achados parecem ir ao encontro dos estudos de Lucchetti et al. (2013), Espinha et al. (2013) e Espíndula, Valle e Bello (2010), mostrando uma espécie de "círculo vicioso" entre falta de treinamento e insegurança de inserir a temática $\mathrm{R} / \mathrm{E}$ na prática clínica.

Como visto no estudo desenvolvido por Lucchetti et al. (2012; 2013), há uma carência de treinamento para habilitar o profissional a lidar com a temática R/E. Os resultados do presente estudo sugerem que o gap entre as expectativas dos estudantes e a realidade do currículo das universidades brasileiras provavelmente acontece não apenas nas faculdades de Medicina, mas na área da saúde como um todo.
As análises de conteúdo mostraram seis categorias que emergiram das entrevistas qualitativas: 1 . Religiosidade e Espiritualidade: fusão de conceitos e conexão com o transcendente; 2. Espiritualidade como facilitadora; 3. Demanda aparece informalmente; 4. Falta de treinamento; 5 . Na prática é diferente; 6 . A temática nos currículos acadêmicos.

\section{Religiosidade e Espiritualidade: Fusão de Conceitos e Conexão com o Transcendente}

A diferenciação de conceitos para os entrevistados nesta pesquisa se dá a partir de preceitos pessoais. De um modo geral, os termos "espiritualidade" e "religiosidade" apareceram amplamente fundidos entre si nas respostas dos participantes e por meio delas pode-se observar que as definições estavam mais próximas do que Koenig (2005) define como uma conexão com o transcendente, como podemos ver no exemplo:

P3: É ter fé, é acreditar né... que tem um ser superior, Deus no caso, e tu acreditando nele tu consegue superar muitas dificuldades... Espiritualidade pra mim é isso, é acreditar, é ter fé em algum ser né.

P1: necessidade né... De crer em algo superior (...)

\section{Espiritualidade como Facilitadora}

Mesmo sem perceber a demanda formal em falar sobre R/E e sem ter passado por um treinamento adequado sobre o tema, a espiritualidade do paciente parece ser entendida como um fator que auxilia a prática em saúde, entre os participantes deste estudo. Os profissionais estão atentos a isso em suas práticas e referem em seus relatos a proximidade da fé com aspectos positivos de personalidade e prognóstico clínico.

P2: Com certeza facilitadora. Eu acho que a pessoa que é mais espiritualizada ela tem uma melhor aceitação.

P6: [...] é uma pessoa mais confiante, mais segura, positiva que o tratamento vai ser eficaz, que vai dar certo.

Nesse sentido, em 2017 o Journal of the American Medical Association (JAMA) (VanderWeele, Balboni, \& Koh, 2017), publicou um viewpoint apresentando estudos longitudinais sobre as relações entre saúde e espiritualidade. Entre outras coisas, o artigo afirma que um foco maior nas questões sobre espiritualidade poderia melhorar as abordagens centradas na pessoa, aumentando o bem-estar há muito procurado por pacientes e médicos. 
De forma prática, Puchalski (2013) desenvolveu uma ferramenta para abordar questões espirituais do sujeito, servindo de guia na identificação de questões de sofrimento espiritual, ou de força por meio dos recursos utilizados para enfrentamento de doenças, por exemplo. $\mathrm{Na}$ Ferramenta de História Espiritual - FICA (Fé, Importância/Influência, Comunidade, Atitude/ Ação), o profissional pode investigar o quanto a fé é importante para seu paciente e o quanto as suas crenças influenciam em sua vida para lidar com o estresse ou decisões difíceis.

Para Alves, Jungues e López (2010) em sua pesquisa com profissionais da saúde, a fé também foi observada como facilitadora e trouxe o caráter curativo provindo de mecanismos internos do ser humano. A influência positiva da fé na saúde foi bastante citada pelos sujeitos entrevistados neste estudo, como seguem os exemplos: P5: Pacientes com fé, com religiosidade tem prognóstico
melhor, uma evolução melhor da sua doença.

P6: Ah, eu acho que sim, pois uma pessoa que costuma ter fé às vezes é até uma pessoa mais positiva.

Kim, Huh e Chae (2015) investigaram a influência da $\mathrm{R} / \mathrm{E}$ durante um período de tratamento de seis meses em pacientes diagnosticados com depressão. $\mathrm{Na}$ análise da pesquisa, considerações subjetivas importantes para a religião e a espiritualidade foram relacionadas à resposta positiva ao tratamento. Dessas variáveis, a espiritualidade permaneceu um preditor significativo no resultado final.

Contudo, muitas vezes, as crenças religiosas/ espirituais também são fonte de sofrimento e conflito, como se observa nos relatos de alguns dos participantes deste estudo. O impacto negativo de tais crenças aparece principalmente relacionado ao comportamento dos pacientes e o entendimento sobre algumas emoções.

P7: Eu vejo que às vežes algumas crenças deixam as pessoas meio confusas em relação a essa questão no que acreditar. Por exemplo, na questão do luto né, da morte, às vezes não tem nada contra a questão do espiritismo, mas, às vezes, isso confunde as pessoas no sentido de deixar a pessoa de deixar ela em sofrimento, pois alguma pessoa disse alguma coisa para ela e como ela se vira com aquilo?

Neste sentido, Koenig (2005) aponta que, mesmo havendo benefícios em alguns pacientes que utilizam a espiritualidade a serviço da sua saúde, o paciente pode dar uma dimensão negativa a espiritualidade. Esse enfoque está associado a piores resultados quando o indivíduo destaca comportamentos como a culpa em relação a si, intolerância e abandono de tratamentos médicos.

Pargament, Tarakeshwar e Hahn (2004) desenvolveu o conceito de "Coping Religioso/Espiritual" para se referir a estratégias de enfrentamento, onde as pessoas utilizam suas crenças religiosas/espirituais em situações de estresse. Para tal, o autor criou a escala Religious Coping Activity Scales (RCOPE) que mede estratégias de coping religioso/espiritual positivas, gerando bem-estar, e negativas, quando a pessoa refere insatisfação na sua relação com o sagrado e ressignifica sua adversidade ou infortúnio como punição ou perseguição.

O Coping Religioso Negativo é um dos fatores que tornam a abertura do profissional às questões religiosas / espirituais de seus pacientes muito importante. Embora não possa tratar dos conflitos enquanto um "religioso", o profissional da saúde deve ser capaz de identificar tais problemas e trabalhar de forma integrada com sacerdotes das diferentes tradições (Moreira-Almeida et al., 2016). E, como afirma um dos entrevistados:

\section{P5: (...) Então seria uma falta de bom senso não utilizar a fé do paciente como instrumento potencializador do meu trata- mento! Então se eu sei que se o paciente orar ele vai melhorar $30 \%$ em relação aos beneficios do tratamento, não usar isso seria falta de sabedoria.}

\section{Demanda Informal ou Implicita}

De uma forma geral, os profissionais percebem que a demanda dos pacientes para falar sobre suas crenças aparece em conversas referidas como "informais" e de uma forma implícita. Para alguns profissionais, o status informal das conversas parece demonstrar que o tema não é relevante ou que não há demanda propriamente dita para tratar sobre religiosidade e/ou espiritualidade.

P2: Eu acredito que não haja uma demanda, acho que de fato, todos os pacientes que nos procuram, talvez por a gente ter esse convivio mais de amizade às vezes, eles acabam sempre falando desse assunto, esse assunto sempre vem (...) Em forma de conversa informal.

P7: Não aparece de uma forma explícita, que eu possa identificar.

A demanda implícita sugere que tanto profissionais quanto pacientes observem a relevância em falar 
em R/E, embora isso seja feito informalmente e num contexto de "amizade" e não de relação de trabalho. Não foram encontrados estudos que apresentassem resultados semelhantes, contudo, a pesquisa de Espíndula et al. (2010) aponta para o receio por parte do profissional, em facilitar uma espécie de "falta de senso de realidade" ao falar explicitamente sobre o assunto com o paciente. Dessa forma, "falar informalmente" pode preencher a necessidade de tocar no assunto (demanda implícita), sem que isso seja integrado ao contexto da prática clínica propriamente dita.

\section{Falta de Treinamento}

A maioria dos participantes referira não estar preparados ou não se sentirem seguros para tratar sobre o tema quando ele aparece nos consultórios por não ter sido treinados para isso:

\section{P6: Nem sempre eu me sinto preparada.}

P4: Totalmente não, porque eu não tive nenhuma capacitação né, a respeito... A minha capacitação é só de curiosidade minha.

Parece ser uma constante entre os profissionais da saúde que observam a temática em suas práticas de trabalho, a busca pessoal pelo tema, como citou o participante 4 (P4). Em um estudo realizado com 120 estudantes de enfermagem da Universidade de São Paulo (2013), os autores investigaram as opiniões desses estudantes sobre a conexão entre os temas saúde, espiritualidade e religiosidade, e também a importância desse assunto na graduação. Quando questionados sobre como buscam informações sobre o tema, 54,6\% dos participantes afirmaram encontrar referência dentro da própria religião. Outros $17,6 \%$ buscam na leitura de livros, $16 \%$ em leitura de artigos científicos, e 27,7\% não buscavam conhecimentos. Isso corrobora a ideia de que, se não há informação e treinamento adequado nos cursos de graduação, os profissionais tendem a buscar conhecimento em suas crenças pessoais o que, pode, na maioria das vezes, ser inadequado.

Nesse mesmo estudo, os participantes referiram que, apesar de observarem a demanda da espiritualidade na prática e, apesar de desejarem manejar o tema de forma satisfatória, não se sentiam seguros para tal. A falta de informação necessária na graduação e o medo de impor as suas próprias crenças para os pacientes dificultam uma abordagem mais efetiva, ampla e integral na área da saúde.
É muito comum, contudo, que em alguns cursos de graduação, como a Psicologia, por exemplo, haja uma diminuição na crença da efetividade da espiritualidade/ religiosidade como algo que possa ajudar o paciente de alguma forma. Em um estudo realizado com estudantes de Psicologia de todo o estado do Rio Grande do Sul (2014), foi notório a diminuição nessa crença e também no coping positivo dos próprios estudantes de Psicologia envolvidos na pesquisa. Uma das hipóteses levantadas foi a de que, no estado do Rio Grande do Sul haja uma predominância pela linha teórica da Psicanálise e esse fato pode ter enviesado os resultados (Cavalheiro \& Falcke, 2014). Não diferente disso, também se obtive a resposta de um dos participantes nesse sentido:

P7: Eu acho que eu tomei, uma certa época, certas teorias como doutrina mesmo... E ai eu substitui a religião pela teoria.

\section{Na Prática é Diferente}

Embora os entrevistados tenham relatado benefícios e conflitos em relação à $\mathrm{R} / \mathrm{E}$ que podem impactar a saúde de seus pacientes, suas falas indicam que "na prática é diferente". Em outras palavras, sugerem que, na prática, não irão abordar essas questões, embora reconheçam que são importantes, sobretudo porque, para eles, remetem a escolhas do aspecto pessoal e privado de seus pacientes:

P2: É um assunto que as pessoas falam, mas num sentido muitas vezes de terem um conforto para elas próprias, do que elas estão passando no momento, do que se importar do que eu penso sobre essa questão.

P6: "não, não tive nada... Nunca teve" porque na graduação não falam sobre isso né... Atépor que isso acaba sendo muito pessoal do profissional buscar... Acho que não é nem do profissional, é do pessoal buscar, men A., de pegar e querer estar bem com a minha questão espiritual.

P1: Eu acho que isso é uma questão só bem individual mesmo. Eu acho que para a minha profissão é importante, mas isso é uma coisa bem pessoal, a espiritualidade!

Também foi possível observar que o profissional não acredita que a sua opinião sobre espiritualidade vai modificar ou influenciar o tratamento ou a confiança do paciente em relação ao seu trabalho:

P2: É um assunto que as pessoas falam, mas num sentido muitas vezes de terem um conforto para elas próprias, do que 
elas estão passando no momento, do que se importar do que eu penso sobre essa questão.

A mesma perspectiva foi encontrada na análise de Alves et al. (2010) sobre a percepção dos profissionais do SUS em relação à espiritualidade e à religiosidade. Nesse estudo, os autores identificaram uma imparcialidade dos sujeitos da pesquisa como a única forma de respeito ao tema. Em consequência disso, pode ser percebido um distanciamento em relação ao assunto principalmente quanto a utilizá-lo como ferramenta. Para os autores, apenas manter-se imparcial e respeitoso sobre a temática espiritualidade/religiosidade, não "ficar falando sobre religião" (p. 434) e não envolver-se com a possibilidade de utilizá-la na prática significa não atender de forma humanizada e não se está considerando o paciente de forma integral, sendo que essas seriam posições relevantes de indivíduos que se dizem promotores da saúde.

\section{A Temática nos Currículos Acadêmicos}

Em sua maioria, os participantes da pesquisa não acham que esse seria um assunto interessante ou viável de discussão no currículo acadêmico:

\begin{abstract}
P6: Acho que tive um semestre com filosofia, ninguém dava bola! E olha que filosofia é algo mais... tipo... estudável assim... Interessante... Imagina colocar uma cadeira com religiosidade ou espiritualidade! Eu não sei se olhariam com bons olhos... E eu não sei se seria eficaz ou se seria bom.
\end{abstract}

P4: Se disser para colocar na grade curricular, ai vai ter resistência de todos!

\section{P2: Eu não sei te dizer se isso é o mais importante.}

Interessante notar que, durante o processo das entrevistas, a espiritualidade sempre foi tida em um sentido positivo tanto para os usuários como na observação dos profissionais. Quando questionados sobre o quanto se sentem preparados para tal abordagem, referiram à falta desse assunto na sua formação. Porém, quando interpelados sobre a possibilidade desse conteúdo na academia, a maioria dos profissionais não acha viável e desconsidera o fator da temática ser relevante nos currículos acadêmicos.

Apesar do acesso sobre o tema da espiritualidade no contexto da saúde não ser uma realidade brasileira, fora do país há uma abertura aparentemente maior para que se tenha acesso ao assunto ainda na formação acadêmica. Em 1993, menos de 5\% das escolas médicas tinham em sua grade curricular uma disciplina que relacionasse o conteúdo da religião/espiritualidade e Medicina, e esse número cresceu para 100 escolas em menos de 15 anos. Seguindo o mesmo movimento, houve um aumento de 59\% das escolas médicas britânicas no acesso a cursos sobre a espiritualidade relacionada com a saúde (Koenig, 2005).

Para Koenig, King e Carson (2012), ressaltar o entendimento científico na temática $\mathrm{R} / \mathrm{E}$ provem $\mathrm{da}$ hipótese de que, na transição entre o século XIX e $\mathrm{XX}$, nomes importantes da Medicina na época disseminaram e defenderam o pressuposto negativo de que experiências espirituais provinham de imaturidade da personalidade, pouca cultura e também decorrentes de alguma neurose ou transtorno mental, e foi constantemente associada à loucura.

Nesse sentido, Dal-Farra e Geremia (2010) propõem três alternativas para que a espiritualidade possa ser incluída nas discussões acadêmicas, como: oferecer diretamente na matriz curricular assuntos que reforcem a importância do tema na prática profissional, também discutem o oferecimento de cursos de extensão e estágios complementares previstos pelas Diretrizes Curriculares Nacionais para a Educação Superior, e a possibilidade de um diálogo transversal onde possa ser discutido a importância do tema para as práticas educativas.

Diante de tudo isso, as análises deste estudo apontam para o que se poderia chamar de "desencontro" entre prática, demanda e formação acadêmica/ profissional. Em outras palavras, há uma contradição quando clínicos observam que o tema surge em conversas informais, que a espiritualidade parece atuar positivamente na saúde de seus pacientes, mas que não possuem treinamento adequado para saber como encaminhar a questão. E vão além: também não julgam possível ou verdadeiramente importante, incluir os temas "religiosidade e espiritualidade" na formação acadêmica. Parece que isso significa: "não temos treinamento para tratar de tais questões, por isso não falamos sobre isso na prática, com nossos pacientes". E, ao mesmo tempo: "mas não queremos realmente receber esse treinamento na academia".

Como entender essas respostas? Como explicar o "círculo vicioso" que se estabelece quando a falta de treinamento gera encaminhamentos informais sobre a temática, insegurança para endereçá-la na prática propriamente dita e crença de que não se pode estudar sobre isso no meio científico e de formação acadêmica? Assim, há garantia de que não haverá inserção da 
temática nas graduações da área da saúde, consolidando o círculo vicioso.

Por outro lado, a insegurança dos profissionais ao afirmarem que "na prática é diferente", também evidencia seu cuidado em não ultrapassar limites éticos dentro de uma temática tão delicada e de âmbito privado. Contudo, a tentativa de manter os "limites" entre questões pessoais e profissionais, nesse caso, parece falhar no momento em que a demanda surge em conversas informais mostrando ao profissional o impacto aparentemente positivo das crenças religiosas/espirituais dos pacientes.

Diante de tais questionamentos, há três diretrizes a serem estabelecidas, fundamentadas em estudos sobre a inserção dos temas R/E na formação profissional e na Declaração da Associação Mundial de Psiquiatria (WPA):

a) A religiosidade e a espiritualidade devem ser investigadas na coleta do histórico do paciente (por meio de uma simples pergunta: "você possui uma religião, religiosidade ou espiritualidade?”). Depois, podem ser seguidas as instruções do questionário FICA ("Qual a importância que sua fé tem para você?" "Você faz parte de uma comunidade ou grupo de pessoas que compartilham a mesma crença?" "Podemos falar abertamente sobre estas questões e auxiliar dentro de nossa proposta de trabalho"); Lembrando que essa abordagem é sempre centrada no significado e no papel (bem como na ausência disso), para o paciente/cliente e nunca para o profissional.

b) Realizar mais estudos, investigando o impacto positivo e negativo da religiosidade e da espiritualidade na saúde, bem como apontando para manejo e conduta clínica mais adequados.

c) Inserir evidências de pesquisa e diretrizes de atendimento relacionados à religiosidade e à espiritualidade em todas as formações da área da saúde.

\section{Considerações Finais}

Esta pesquisa teve como principal objetivo observar as percepções dos profissionais de saúde em relação aos temas "religiosidade" e "espiritualidade" e sua inserção na prática clínica. A relevância deste estudo foi tratar abertamente de questões que não costumam aparecer entre as principais diretrizes e orientações profissionais, embora uma série de estudos mostre seu impacto na saúde dos pacientes e clientes que buscam atendimento clínico.

A pesquisa teve como principal resultado, a observação de uma espécie de "círculo vicioso" entre falta de treinamento sobre $\mathrm{R} / \mathrm{E}$ na prática clínica; demanda implícita e encaminhamento informal quando a temática surge; insegurança em tratar da temática de forma ética e estritamente profissional, gerando receio em trazer o tema para as formações e treinamentos de profissionais da área da saúde. Resumidamente: falta de informação e treinamento sobre $\mathrm{R} / \mathrm{E}$ na prática clínica gera mais falta de informação e treinamento sobre $\mathrm{R} / \mathrm{E}$ na prática clínica.

Importante lembrar que esse "círculo vicioso" foi observado em um recorte qualitativo de uma amostra pequena de profissionais de diferentes áreas da saúde, limitando a generalização dos resultados. Assim sendo, futuros estudos poderão investigar amostras maiores (com abordagens quantitativas) ou, mesmo, amostras de profissionais de áreas específicas da saúde (com abordagens qualitativas), a fim de observar se serão encontrados resultados semelhantes.

\section{Referências}

Alminhana, L. O., Ramos, T. P. T., Nardini, M., \& Marques, L. F. (2017). Saúde e doença nas experiências religiosas/espirituais: Integrando modelos de Wilber e Cloninger. PsicoUSF. Braçança Paulista, SP. Vol. 22, n. 2 (maio./ago. 2017), p. 351-360. doi: 10.1590/1413-82712017220213.

Alves, J. de S., Junges, J. R., \& López, L. C. (2010). A dimensão religiosa dos usuários na prática do atendimento à saúde: Percepção dos profissionais da saúde. Mundo saúde (Impr.), 430-436.

Arruda, M. M., Marafanti, I., da Silva, M. E., Pinheiro, M. C. P., Shiozawa, P., \& Cordeiro, Q. (2018). Investigação dos aspectos bioéticos envolvidos na assistência religiosa a pacientes com transtornos mentais graves internados em um hospital psiquiátrico. Arquivos Médicos dos Hospitais e da Faculdade de Ciências Médicas da Santa Casa de São Paulo, 58(3), 113-120. Recuperado de http:// arquivosmedicos.fcmsantacasasp.edu.br/index. $\mathrm{php} / \mathrm{AMSCSP} /$ article/view/23

Bardin, L. (2008). Análise de Conteúdo (5 edição). Edições 70. Lisboa. 
Campos, A. F., \& Ribeiro, J. P. (2017). Psicoterapia e espiritualidade: Da gestalt-terapia à pesquisa contemporânea. Revista da Abordagem Gestáltica, 23(2), 211-218.

Cavalheiro, C. M. F., \& Falcke, D. (2014). Espiritualidade na formação acadêmica em psicologia no Rio Grande do Sul. Estud. Psicol. 31(1), 35-44.

Dal-Farra, R. A., \& Geremia, C. (2010). Educação em saúde e espiritualidade: Proposições metodológicas. Rev. bras. educ. méd, 34(4), 587-597.

Dalgalarrondo, P. (2007). Brazilian studies on religion and mental health: history and current perspectives. Archives of Clinical Psychiatry. 34, 25-33. doi: 10.1590/S0101-60832007000700005

Dein, S., Cook, C. C. H., Powell, A., \& Eagger, S. (2010). Religion, spirituality and mental health. The Psychiatrist, 34(2), 63-64. doi: 10.1192/pb.bp.109.025924

Espíndula, J. A., Valle, E. R. M. D., \& Bello, A. A. (2010). Religion and Spirituality: The Perspective of Health Professionals. Revista Latino-Americana de Enfermagem, 18(6), 1229-1236. doi: 10.1590/ S0104-11692010000600025

Espinha, D. C. M., Camargo, S. M. de, Silva, S. P. Z., Pavelqueires, S., \& Lucchetti, G. (2013). Opinião dos estudantes de enfermagem sobre saúde, espiritualidade e religiosidade. Revista Gaúcha de Enfermagem, 34(4), 98-106. doi: 10.1590/ S1983-14472013000400013

Farias, M.,Underwood, R., \&Claridge, G. (2013). Unusual but sound minds: Mental health indicators in spiritual individuals. British Journal of Psychology, 104(3), 364-381. doi: 10.1111/j.2044-8295.2012.02128.x

Kim, N.-Y., Huh, H.-J., \& Chae, J.-H. (2015). Effects of religiosity and spirituality on the treatment response in patients with depressive disorders. Comprehensive Psychiatry, 60, 26-34. doi: 10.1016/j. comppsych.2015.04.009

Koenig, H. (2005). A espiritualidade no cuidado com o paciente: Por que, quando, como e o que. São Paulo: FE.

Koenig, H., King, D., \& Carson, V., B. (2012). Handbook Of Religion and Health. New York: Oxford University Press.

Kørup, A. K., Søndergaard, J., Christensen, R. dePont, Nielsen, C. T., Lucchetti, G., Ramakrishnan, P., ... Hvidt, N. C. (2018). Religious Values in Clinical
Practice are Here to Stay. Journal of Religion and Health. doi: 10.1007/s10943-018-0715-y

Lavorato-Neto, G., Rodrigues, L., Turato, E. R., \& Campos, C. J. G. (2018). The free spirit: spiritualism meanings by a Nursing team on psychiatry. Rev Bras Enferm, 71(2), 280-288.

Lucchetti, A. L. G., Peres, M. F. P., Vallada, H. P., \& Lucchetti, G. (2015). Spiritual Treatment for Depression in Brazil: An Experience From Spiritism. EXPLORE: The Journal of Science and Healing, 11(5), 377-386. doi: 10.1016/j.explore.2015.07.002

Lucchetti, G., de Oliveira, L. R., Koenig, H. G., Leite, J. R., \& Lucchetti, A. L. (2013). Medical students, spirituality and religiosity-results from the multicenter study SBRAME. BMC Medical Education, 13, 162. doi: 10.1186/1472-6920-13-162

Lucchetti, G., Granero, A. L., Bassi, R. M., Latorraca, R., \& Nacif, S. A. da P. (2010). Espiritualidade na prática clínica: O que o clínico deve saber? Rev. Soc. Bras. Clín. Méd, 8(2). Recuperado de http://files. bvs.br/upload/S/1679-1010/2010/v8n2/a012. pdf

Lucchetti, G., Lucchetti, A. L. G., Espinha, D. C. M., de Oliveira, L. R., Leite, J. R., \& Koenig, H. G. (2012). Spirituality and health in the curricula of medical schools in Brazil. BMC Medical Education, 12, 78. doi: 10.1186/1472-6920-12-78

Machado, F. R., Zangari, W., de Oliveira Maraldi, E., Martins, L. B., \& Shimabucuro, A. H. (2017). Contribuições da psicologia para a compreensão das relações entre a espiritualidade, a religiosidade e as experiências anômalas. Clareira-Revista de Filosofia da Região Amazônica, 3(2), 2-21. Recuperado de http://www.revistaclareira.com.br/index.php/ clareira/article/view/114

Martins, L. B., \& Zangari, W. (2012). Relations among typical contemporary anomalous experiences, mental disorders and spiritual experiences. Archives of Clinical Psychiatry. 39(6), 198-202. doi: 10.1590/ S0101-60832012000600004

Menezes Jr., A., Alminhana, L., \& Moreira-Almeida, A. (2012). Sociodemographic and anomalous experiences profile in subjects with psychotic and dissociative experiences in religious groups. $A r$ chives of Clinical Psychiatry . 39(6), 203-207. doi: 10.1590/S0101-60832012000600005 
Moreira-Almeida, A., Neto, F. L., \& Greyson, B. (2007). Dissociative and Psychotic Experiences in Brazilian Spiritist Mediums. Psychotherapy and Psychosomatics, 76(1), 57-58. doi: 10.1159/000096365

Moreira-Almeida, A., Pinsky, I., Zaleski, M., \& Laranjeira, R. (2010). Religious involvement and sociodemographic factors: a Brazilian national survey. Archives of Clinical Psychiatry (São Paulo), 37(1), 12-15. doi: 10.1590/S0101-60832010000100003

Moreira-Almeida, A., Sharma, A., van Rensburg, B. J., Verhagen, P. J., \& Cook, C. C. H. (2016). WPA Position Statement on Spirituality and Religion in Psychiatry. World Psychiatry, 15(1), 87-88. doi: 10.1002/wps.20304

Nepomuceno, F. C. L., Almeida, D. A. de, Fonseca, R. C., \& Vieira, R. da S. (2018). Impactos da Espiritualidade na Saúde do Idoso: Relatos dos Atendimentos da Fisioterapia. Cadernos de Educação, Saúde e Fisioterapia, 5(10). Recuperado de http://revista.redeunida.org.br/ojs/index. $\mathrm{php} /$ cadernos-educacao-saude-fisioter/article/ view/1903

Neto, M., \& Paiva, A. de. (2016). Percepção da espiritualidade por fisioterapeutas em uma UTI: Uma pesquisa qualitativa. Recuperado de http:/ /dspace. est.edu.br:8080/jspui/handle/BR-SlFE/659

Oman, D., \& Lukoff, D. (2018). Mental Health, Religion, and Spirituality. In Why Religion and Spirituality Matter for Public Health (pp. 225-243). Springer, Cham.

Pargament, K. I., Koenig, H. G., \& Perez, L. M. (2000). The many methods of religious coping: Development and initial validation of the RCOPE. Journal of Clinical Psychology, 56(4), 519-543. doi: 10.1002/ (SICI)1097-4679(200004)56:4<519::AIDJCLP6>3.0.CO;2-1

Pargament, K. I., Koenig, H. G., Tarakeshwar, N., \& Hahn, J. (2004). Religious Coping Methods as Predictors of Psychological, Physical and Spiritual Outcomes among Medically Ill Elderly Patients: A Two-year Longitudinal Study. Journal of Health Psychology, 9(6), 713-730. doi: 10.1177/1359105304045366
Pargament, K. I., Smith, B. W., Koenig, H. G., \& Perez, L. (1998). Patterns of Positive and Negative Religious Coping with Major Life Stressors. Journal for the Scientific Study of Religion, 37(4), 710-724. doi: $10.2307 / 1388152$

Parra, A., \& Espinosa Paul, L. (2010). Comparación entre la Esquizotipia Positiva y Perturbadora con la Espiritualidad y las Experiencias Paranormales en Población No-Clínica. Revista Argentina de Clinica Psicológica, XIX(2).

Puchalski, C. M. (2001). The role of spirituality in health care. Proceedings (Baylor University. Medical Center), 14(4), 352-357.

Puchalski, C. M. (2013). The FICA Spiritual History Tool \#274. Journal of Palliative Medicine, 17(1), 105106. doi: 10.1089/jpm.2013.9458

Saad, M., Masiero, D., \& Battistella, L. R. (2016). Espiritualidade baseada em evidências. Acta Fisiátrica, 8(3), 107-112. doi: 10.5935/0104-7795.20010003

Siqueira, H. C. H. D., Cecagno, D., Medeiros, A. C. D., Sampaio, A. D., \& Rangel, R. F. (2017). Espiritualidade no processo saúde-doença-cuidado do usuário oncológico: Olhar do enfermeiro. Rev. enferm. UFPE online, 11(8), 2996-3004. doi: 10.5205/reuol.11064-98681-4-ED.1108201702.

Simões, N. D., Martins, P. G., dos Santos, R. O. P., Santana, F. R., \& Pilger, C. (2018). Espiritualidade e saúde: Experiência de uma disciplina na graduação de enfermagem. Revista de Enfermagem da UFSM, 8(1), 181-191. doi: 10.5902/2179769225038

VanderWeele, T. J., Balboni, M. J., \& Balboni, T. A. (2018). The Initiative on Health, Religion and Spirituality at Harvard: From Research to Education. In Why Religion and Spirituality Matter for Public Health (pp. 371-382). Springer, Cham.

VanderWeele, T. J., Balboni, T. A., \& Koh, H. K. (2017). Health and Spirituality. JAMA, 318(6), 519-520. doi: 10.1001/jama.2017.8136

Recebido em: 09/04/2018 Reformulado em: 10/07/2018; 20/10/2018 Aprovado em: 07/12/2018 
Sobre as autoras:

Juliana Schmidt Raddatz é psicóloga clínica, mestranda em Psicologia Clínica - PUCRS, e sócia-fundadora do instituto Unusual in Psychology RS. RADDATZ, Juliana. S

E-mail: Raddatz.ju@gmail.com

ORCID: https://orcid.org/0000-0001-8024-511X

Roberta Fin Motta é doutora em Psicologia, professora do curso de Psicologia da Pontifícia Universidade Católica do Rio Grande do Sul (PUCRS). MOTTA, Roberta. F.

E-mail: robertafmotta@gmail.com

ORCID: https://orcid.org/0000-0003-4074-0161

Letícia Oliveira Alminhana é pesquisadora colaboradora no Laboratório de Estudos Avançados Multidisciplinares (LEAM), vinculado à Pró-reitoria de Pós-graduação da UERGS e diretora do Instituto Unusual in Psychology/RS. Recentemente finalizou 5 anos como professora colaboradora (PNPD/CAPES) no Programa de Pós-Graduação em Psicologia da PUCRS, possui pós-doutorado pela University of Oxford/UK, e doutorado em Saúde pela UFJF/MG. E-mail: leticiaalminhana@gmail.com

ORCID: https://orcid.org/0000-0001-8829-4493

Contato com as autoras:

Av. Getúlio Vargas, 901, sala 1506

Porto Alegre-RS, Brasil

CEP: 90150-000 